\title{
The Kinematics and Eye Movements for a Two-Eyed Robot Head
}

\author{
Fenglei Du Michael Brady \\ Robotics Research Group \\ Engineering Science Department \\ Oxford University \\ 19 Parks Road Oxford OX1 3PJ
}

\begin{abstract}
This paper is part of a study of gaze control for a two-eyed robot head. The kinematics of the head and eye movement planning for the subcontrols loosely corresponding to saccade, pursuit, VOR(vestibulo-ocular reflex), OKR(optokinetic reflex), and vergence are presented.
\end{abstract}

\section{Kinematics}

The gross structure of the robot head and the coordinate frames are shown in figure 1. There are four degrees of freedom, i.e., pan, tilt, and left and right vergence joint. In the figure, $P$ is the fixation point. $\theta_{h}$ is the pan angle. The inter-ocular separation is $B$. The ray from ${ }^{e} O$ to $P$ makes gaze angle $\alpha$ with ${ }^{e} X . \theta_{r}$ and $\theta_{l}$ are the vergence joint angles. $a$ is the distance between origin of $e$ (eye) frame and $s$ (system) frame. The tilt angle is denoted $\theta_{t}$. Assume that the fixation point position and its velocity vector are ${ }^{s} X_{a}$ and ${ }^{s} V_{a}$ in $s$ frame, we find:

$$
{ }^{s} X_{a}=\mathrm{F}_{1}(\Theta) ;{ }^{s} V_{a}=J(\Theta) * \Omega
$$

where $F_{1}(\cdot)$ is a set of nonlinear functions, $\Theta=\left\{\theta_{r}, \theta_{l}, \theta_{h}, \theta_{t}\right\}^{T}$ is the joint position vector of the head, $\Omega=\left\{\omega_{h}, \omega_{t}, \omega_{r}, \omega_{l}\right\}$ is the joint velocity vector of the head, and $J$ is the $3 \times 4$ Jacobian matrix,
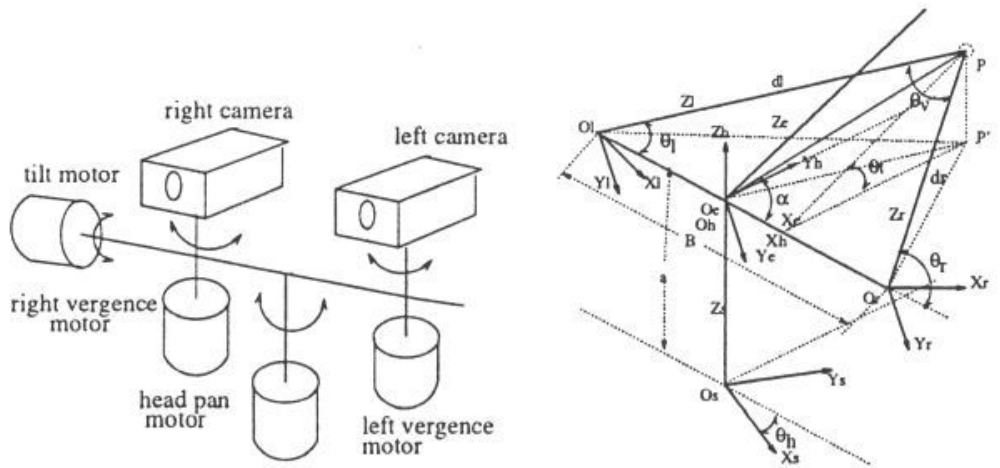

Figure 1: The structure of the robot head and its coordinate frames 


\section{Inverse Kinematics}

If we want the head to fixate on the object at ${ }^{s} X$, i.e., to maintain the image of the object at the center of the left and right image planes, we obtain only three constraints. For our robot head, in order to obtain unique solution, further constraint must be added. If we propose to maintain the eyes in their most "comfortable" positions. We find:

$$
\Theta=\mathbf{F}_{2}\left({ }^{s} X\right)
$$

If the head pan angle $\theta_{h}$ is held stationary for a new fixation, we obtain: $\left(\Theta_{1}\right.$ is composed of the last three components of $\Theta$ ):

$$
\Theta_{1}=\mathbf{F}_{3}\left({ }^{s} X, \theta_{h}\right)
$$

Suppose that the velocity of the object of interest in the system frame is ${ }^{s} V$. If we want to track it, the following relationships must hold $\left(\mathrm{J}^{+}\right.$is the pseudoinverse of the Jacobian matrix):

$$
\Omega=J^{+} *^{s} V
$$

\section{Eye movement planning}

\section{$3.1 \quad$ Vergence}

Suppose that the relative retinal disparity of the image of the object in the $X$ direction of the image plane is $\mu_{x}$. If the system tries to keep the "eyes" in their most "comfortable" position, vergence planning process is listed in table 1 .

Table 1: The vergence planning and suitable conditions

\begin{tabular}{|l|l|l|}
\hline \hline scheme & planning & condition \\
\hline changing left & $\delta \theta_{l}=\frac{\mu_{x}}{f_{l}}$ & $\left(\alpha>90^{\circ}\right.$ and $\left.\mu<0\right)$ or $\left(\alpha<90^{\circ}\right.$ and $\left.\mu>0\right)$ \\
\hline changing right & $\delta \theta_{r}=-\frac{\mu_{x}}{f_{r}}$ & $\left(\alpha>90^{\circ}\right.$ and $\left.\mu>0\right)$ or $\left(\alpha<90^{\circ}\right.$ and $\left.\mu<0\right)$ \\
\hline changing both & $\delta \theta_{r}=-\frac{\mu_{x}}{2 f_{r}}$ & gaze angle is approximately $90^{\circ}$ \\
& $\delta \theta_{l}=\frac{\mu_{x}}{2 f_{l}}$ & \\
\hline \hline
\end{tabular}

\subsection{Saccade}

Assume that the new point of interest is ${ }^{s} X$, the desired state of the head to achieve a saccade can be computed from equation 2 or 3 . There are an infinite number of solutions according to the different head pan positions. Among these, three are most interesting: (a) keeping the head pan angle fixed saccade (using motion other than head pan to achieve the saccade); (b) keeping gaze angle $90^{\circ}$ saccade (maintaining the gaze angle $90^{\circ}$ during a saccade); (c) full motion saccade (using all the joints simultaneously to achieve a saccade). Saccade must take care of three situations: (i) simple saccade. The head makes a saccade from a stationary state to a stationary object. (ii) saccade followed by tracking. (iii) saccade after tracking. 


\subsubsection{Simple Saccade}

For keeping the head pan angle fixed saccade and keeping gaze angle $90^{\circ}$ saccade, we can obtain the motion vector use equations 2 or 3 . Using bang-bang or bang-coastbang motion, the saccade can easily be planned. For a full motion saccade, path planning is more complicated.

For $\theta_{h} \in\left\{\theta_{h_{-} \text {now }}, \theta_{h}^{d}\right\}$ or if $\theta_{h_{-} \text {now }}>\theta_{h}^{d}, \theta_{h} \in\left\{\theta_{h}^{d}, \theta_{h_{-} \text {now }}\right\}$, from equation 3 we can obtain:

$$
\theta_{t}=f_{1}\left(\theta_{h},{ }^{s} X\right) ; \quad \theta_{l}=f_{2}\left(\theta_{h},{ }^{s} X\right) ; \quad \theta_{r}=f_{3}\left(\theta_{h},{ }^{s} X\right)
$$

where ${ }^{s} X$ is the target of the saccade and $f_{i}$ is a nonlinear function. Assume that the motion time for the head pan is $t_{h}$ for a $\theta_{h}$ and the corresponding motion time for other joints are $t_{t}, t_{l}, t_{\tau}$ respectively. The above equations can be rewritten as:

$$
t_{t}=F_{1}\left(t_{h},{ }^{s} X\right) ; \quad t_{l}=F_{2}\left(t_{h},{ }^{s} X\right) ; \quad t_{r}=F_{3}\left(t_{h},{ }^{s} X\right)
$$

These equations are highly nonlinear, so optimal search for a minimum time saccade is difficult. We use instead a suboptimal solution, see [2]:

\subsubsection{Saccade followed by tracking}

Assume that the position and the velocity of the object of interest are ${ }^{s} X$ and ${ }^{s} V$, and that the fixation point is ${ }^{s} X_{a}$. If the time required to complete the saccade is $\delta t$, the desired velocity of fixation point should be: ${ }^{s} V_{d}={ }^{s} V+\frac{{ }^{s} X-{ }^{s} X_{a}}{\delta t}$. Using equation 1 , this saccade can be planned. In real system, because of the maximum velocity limits of the joints, above equation is used only to find a motion direction which can reduce the position error.

\subsubsection{Saccade after Tracking}

This is the same as the simple saccade except that the initial joint speed of the head is not zero. It can easily be planned use the similar method as simple saccade, see [2].

\section{$3.3 \quad$ VOR}

In our approach, VOR is redefined as the process of adjusting the head pan angle so that the "eyes" can maintain their most "comfortable" position, i.e., to keep the gaze angle near to $90^{\circ}$. Assume that the current pan angle is $\theta_{h}^{\circ}$, and that the pan angle required to achieve $90^{\circ}$ gaze angle is $\theta_{h}^{d}$. The head pan speed to achieve VOR can be given by:

$$
\omega_{h}=k_{h} *\left(\theta_{h}^{d}-\theta_{h}^{0}\right)
$$

To reduce the difficulties of implementation, this equation can be replaced by:

$$
\omega_{h}=k_{h} *\left(\theta_{l}^{0}+\theta_{r}^{0}-180^{\circ}\right)
$$

where $k_{h}$ is a constant. Known $\omega_{h}$, VOR planning is straightforward using the planning method discussed in section 3.4.4. 


\subsection{Pursuit}

Assume that the object of interest is at ${ }^{s} X$ and moving with velocity ${ }^{s} V$. The position of the fixation point is ${ }^{s} X_{a}$. For the head to track the object, the velocity of the fixation point must satisfy: ${ }^{s} V_{d}={ }^{s} V+\frac{{ }^{s} X-{ }^{s} X_{a}}{\delta t}$ ( $\delta t$ : sample time). Using different constraints, different pursuit schemes can be planned.

\subsubsection{Full motion tracking}

If we want to use all the joint simultaneously to achieve tracking, equation 1 can be used to planned the motion.

\subsubsection{Tracking while holding the head pan angle fixed}

If the movement of joints other than head pan are used to achieve tracking, $\omega_{h}$ would always be zero during tracking. It follows:

$$
\Omega_{1}^{d}=J_{1}(\Theta)^{-1} *^{s} V_{d}
$$

where $\Omega_{1}^{d}=\left[\omega_{t}, \omega_{l}, \omega_{r}\right]^{T}$ and $J_{1}(\Theta)$ is $3 \times 3$ submatrix of $J(\Theta)$.

\subsubsection{Tracking while holding the gaze angle equal to $90^{\circ}$}

If we want the gaze angle is $90^{\circ}$ during tracking, the motion of left and right vergence joint must satisfy: $\omega_{l}=-\omega_{r}$. It follows:

$$
\Omega_{2}^{d}=J_{2}(\Theta)^{-1} *^{s} V_{d}
$$

where $\Omega_{2}^{d}=\left[\omega_{h}, \omega_{t}, \omega_{l}\right]^{T}$ and $J_{2}(\Theta)$ is the $3 \times 3$ submatrix computed from $J(\Theta)$.

\subsubsection{Tracking with VOR}

Suppose that in order to achieve a VOR we want the head to pan with speed $\omega_{h}$, then the following relationship must hold $\left(J_{h}\right.$ is the first column of $\left.J(\Theta)\right)$ :

$$
\Omega_{1}^{d}=J_{1}(\Theta)^{-1} *\left({ }^{s} V_{d}-\omega_{h} * J_{h}\right)
$$

\subsection{Visual Correction}

For saccade, if the position of the object of interest is known inexactly, the visual correction and VOR can be combined as ( $K_{p / v}$ :gain matrix):

$$
\Omega_{1}=J_{1}^{-1} *\left(K_{p} *\left({ }^{s} X-{ }^{s} X_{a}\right)+K_{v} *\left({ }^{s} V-{ }^{s} V_{a}\right)-\omega_{h} * J_{h}\right)
$$

\section{References}

[1] F. Du, M. Brady, D. Murray. Gaze control for a two-eyed robot head. In Proceeding of British Machine Vision Conference 91, Glasgow, Sep. 1991.

[2] Fenglei Du. The Fundamentals for a two-eyed active vision system. First Year Report, Robot Research Group, Oxford University, 1991. 